\title{
Active Nuclear Material Detection And Imaging
}

\section{IEEE Nuclear Science Symposium}

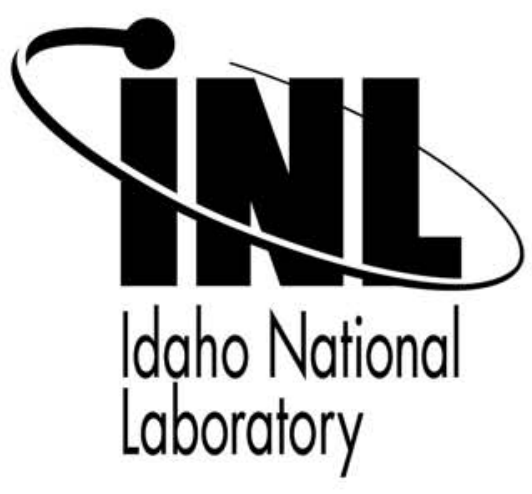

This is a preprint of a paper intended for publication in a journal or proceedings. Since changes may not be made before publication, this preprint should not be cited or reproduced without permission of the author. This document was prepared as an account of work sponsored by an agency of the United States Government. Neither the United States Government nor any agency thereof, or any of their employees, makes any warranty, expressed or implied, or assumes any legal liability or responsibility for any third party's use, or the results of such use, of any information, apparatus, product or process disclosed in this report, or represents that its use by such third party would not infringe privately owned rights. The views expressed in this paper are not necessarily those of the United States Government or the sponsoring agency. 


\title{
Active Nuclear Material Detection and Imaging
}

\author{
Daren R. Norman, James L. Jones, Kevin J. Haskell, Peter E. Vanier, Leon Forman
}

\begin{abstract}
An experimental evaluation has been conducted to assess the operational performance of a coded-aperture, thermal neutron imaging system and its detection and imaging capability for shielded nuclear material in pulsed photonuclear environments. This evaluation used an imaging system developed by Brookhaven National Laboratory. The active photonuclear environment was produced by an operationallyflexible, Idaho National Laboratory (INL) pulsed electron accelerator. The neutron environments were monitored using INL photonuclear neutron detectors. Results include experimental images, operational imaging system assessments and recommendations that would enhance nuclear material detection and imaging performance.
\end{abstract}

\section{INTRODUCTION}

$\mathrm{E}^{\mathrm{n}}$ nhanced detection and imaging capabilities of nuclear ${ }_{\text {material is important for nonproliferation. A thermal }}$ neutron imaging system was operated in pulsed photonuclear environments to enhance its capability to detect and image nuclear material. Fig. 1 shows an image developed from photonuclear induced delayed neutron emissions from depleted uranium (DU) in polyethylene. This image would not be possible without the pulsed photonuclear environment. This active environment results in photofission interactions in nuclear materials. After the nuclear material photofissions, delayed neutrons are emitted. These delayed neutrons can provide several orders-of-magnitude more neutrons than is naturally emitted from the nuclear material. This active photonuclear technique greatly enhances the detection and imaging capability of the thermal neutron imaging system.

While the photonuclear stimulation provides for nuclear material detection and imaging, there are two major challenges that must be overcome. The first is to recover from the photon flash which occurs each time the accelerator pulses. This is especially true for the imaging system because it was design to operate in a passive environment. The second challenge is to differentiate between the photoneutrons that are produced during the photon flash and the delayed neutrons produced from the nuclear material. To better understand how the pulsed photonuclear detection and imaging technique works for nuclear material, the active pulsed photonuclear environment, imaging system, experimental setup, and

Manuscript received November 11, 2005.

This work was supported in part by the U.S. Department of Energy Office of Nonproliferation and National Security under Contract Number DE-AC07$05 \mathrm{ID} 14517$.

D. R. Norman, J. L. Jones, and K. J. Haskell are with the Idaho National Laboratory, Idaho Falls, ID 83415 USA (telephone: 208-526-3953, e-mail: Daren.Norman@inl.gov).

P. E. Vanier, and L. Forman are with Brookhaven National Laboratory, Upton, NY 11973 USA (telephone: 631-344-3535, e-mail: vanier@bnl.gov). resulting images will be discussed. Also, recommendations that may further enhance the detection and imaging capabilities of the system in pulsed photonuclear environments are provided.

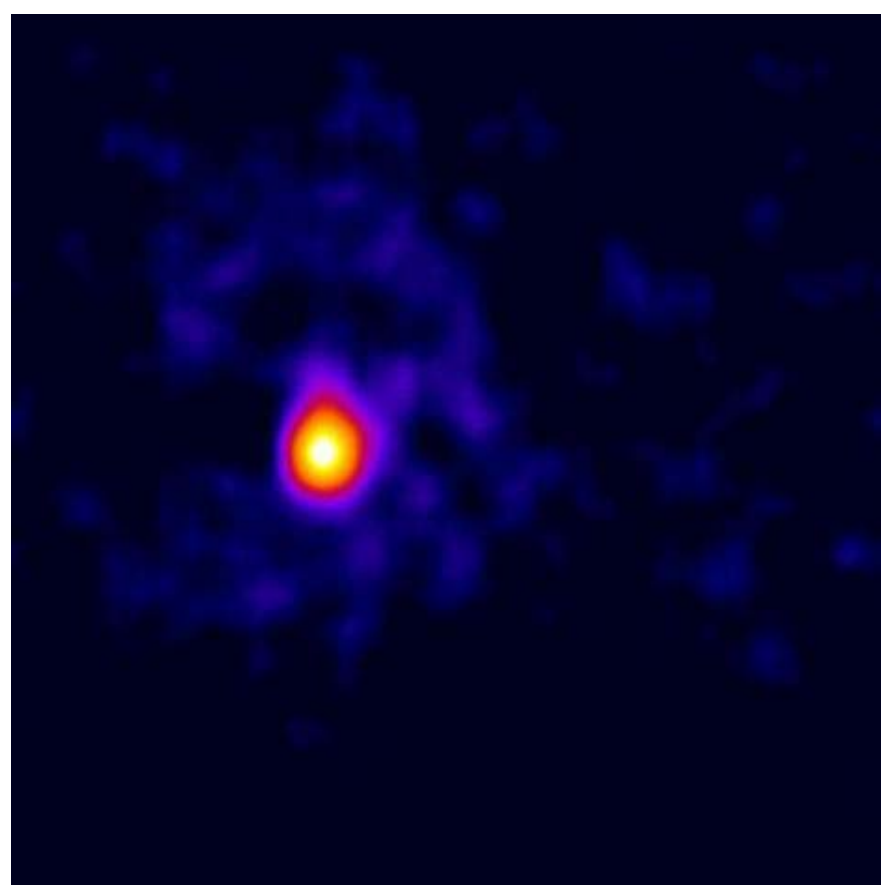

Fig. 1 An image of 5-kilograms of DU delevoped from the emission of photofission-induced, delayed neutrons with the thermal neutron imaging system. For this image the DU to detector distance was 2 meters and provided an image space of about a 1 meter $^{2}$ at the target. This image of the DU in a polyethylene target was processed without binning, and combining the data from "mask" and "antimask" acquisitions.

\section{ACTIVE ENVIRONMENT}

The active environment selected for these tests use energetic photons generated by an electron accelerator. The photons for each pulse are only produced for a short time (few microseconds) regardless of the time between pulses. The time between pulses can be varied to maximize the detection efficiency. These photons can stimulate nuclei to induce photonuclear reactions in most materials. Photonuclear reactions have threshold energies starting at about $2-\mathrm{MeV}$ for hydrogen and beryllium, but greater than $5-\mathrm{MeV}$ for most other materials. The cross sections for photonuclear reactions generally have a broad peak between 10 - and $30-\mathrm{MeV}$ with values ranging from about 10-millibarns to several hundred millibarns. This region is often referred to as the giant dipole resonance region. A photonuclear reaction of particular interest is photofission. Photofission only occurs in nuclear materials and produces fission fragments which can produce 
delayed neutrons as depicted in Fig. 2. These reactions can produce copious amounts of neutrons during the photon pulse. These photoneutrons, created during the pulse, cause interference when trying to detect nuclear material. The neutrons that are generated during the photon pulse dies-away after several milliseconds leaving only delayed neutrons from photofission, if nuclear material is present.
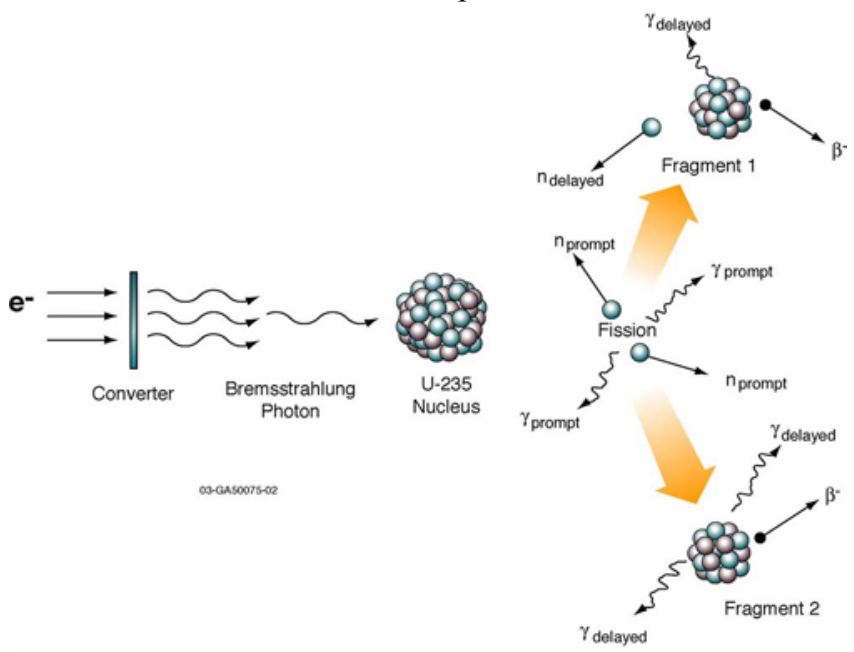

Fig. 2. Graphical depiction of the photofission process for Uranium-235.

We used the INL Varitron 2- to $12-\mathrm{MeV}$ selectable energy electron linac [1] to generate the photonuclear environments. The Varitron was utilized with a nominal 5- and $10-\mathrm{MeV}$ operation, at pulse rates of 50 and 125 pulses per second. The INL Photonuclear Neutron Detector [2] (PND) and a 10atmosphere unmoderated helium-3 (bare He-3) detector were used to monitor the delayed and thermal neutrons. Fig. 3 shows the time-dependent response of these detectors with DU shown by the red plots and with the DU replaced with tungsten (W) shown by the green plots. As demonstrated in Fig. 3, the "room return" thermal neutrons (No DU) have died-away sufficiently such that the DU delayed neutrons provide more of the detected signal after about seven milliseconds than the "room return" neutrons.

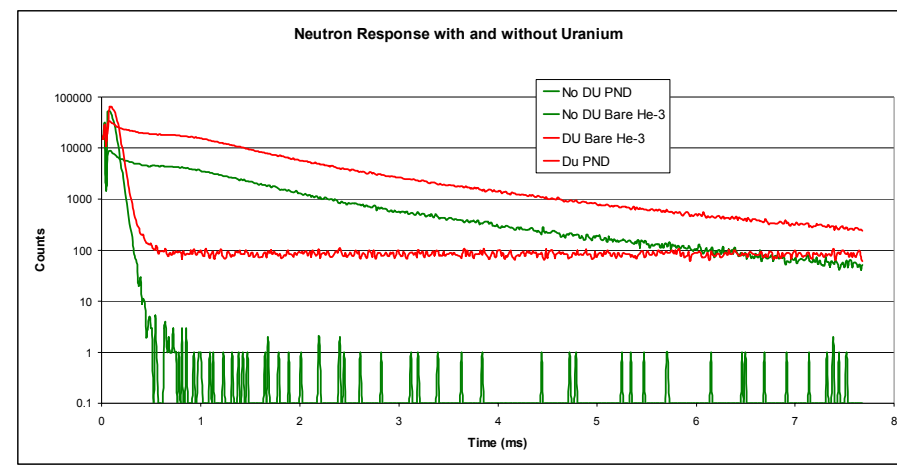

Fig. 3. Time-dependent thermal and delayed photofission neutron responses for PND and bare Helium-3 detectors using a $10-\mathrm{MeV}$ linac operation at a pulse rate of 125 pulses per second.

\section{IMAGING SYSTEM}

The Brookhaven National Laboratory (BNL) coded aperture thermal neutron imaging system [3] was utilized for this assessment. This imaging system uses a 6-atmosphere, bare He-3 chamber as the neutron detector and, therefore, should have a similar time response as the INL bare He-3 neutron detector. The imaging system shown in Fig. 4 has a 2dimensional grid ( 20 by 17 centimeters) inside the cylindrical stainless steel chamber. The coded aperture is several centimeters in front of the detector chamber.

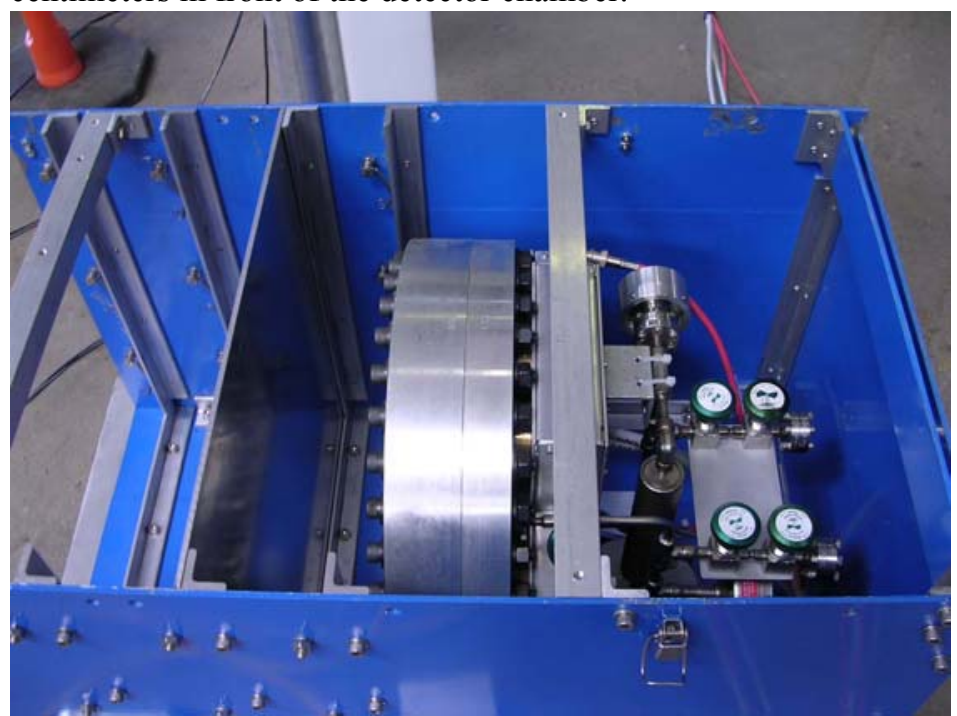

Fig. 4. The internal components of the BNL coded aperture thermal neutron imaging system.

Different apertures can be used with the imaging system at different distances from the chamber to provide different resolution and fields of view respectably. The coded aperture was a 19x19 cadmium, high-precision-modified, uniformlyredundant array used to mask thermal neutrons. Fig. 5 shows the imaging system for one of the test configurations. (Note the coded aperture.)

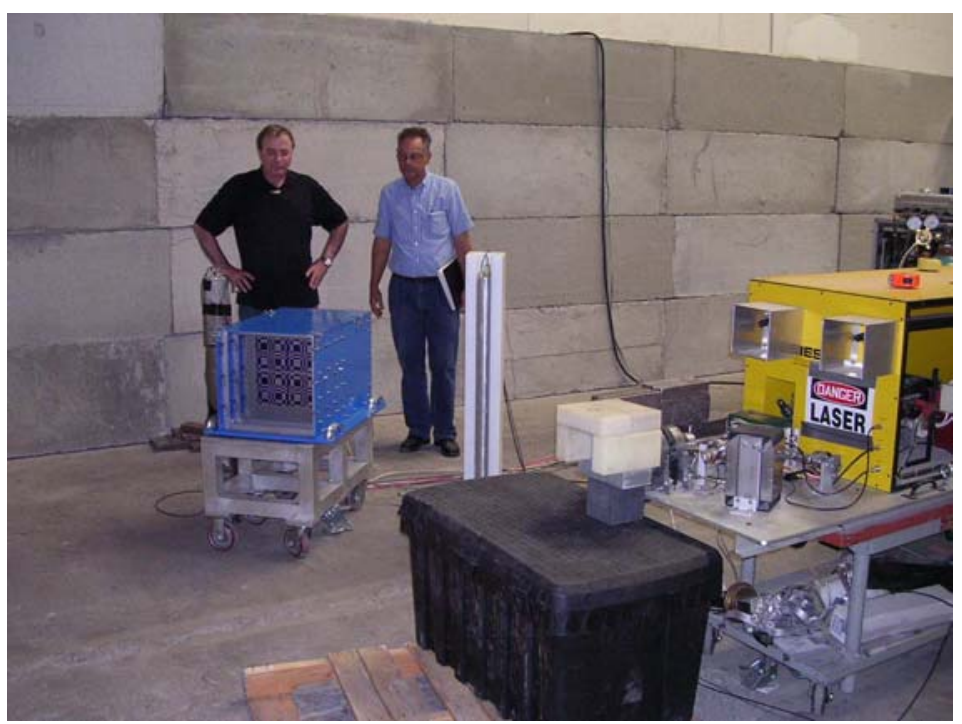

Fig. 5 BNL imaging system and the basic test configuration. The linac is on the right side. 


\section{EXPERIMENTAL SETUP}

The general experimental setup is shown in Fig. 6 and consisted of the electron accelerator (i.e. Varitron), shielding for the side of the Varitron, imaging system, several pieces of polyethylene, various heavy metals, and 5-kilograms of depleted uranium. The imaging system was shielded with at least 10 centimeters of high $\mathrm{Z}$ material at the electron-tophoton converter. The converter was 1 meter away from the target area where the DU or W would be placed. The imaging system was 2 meters from the target area.

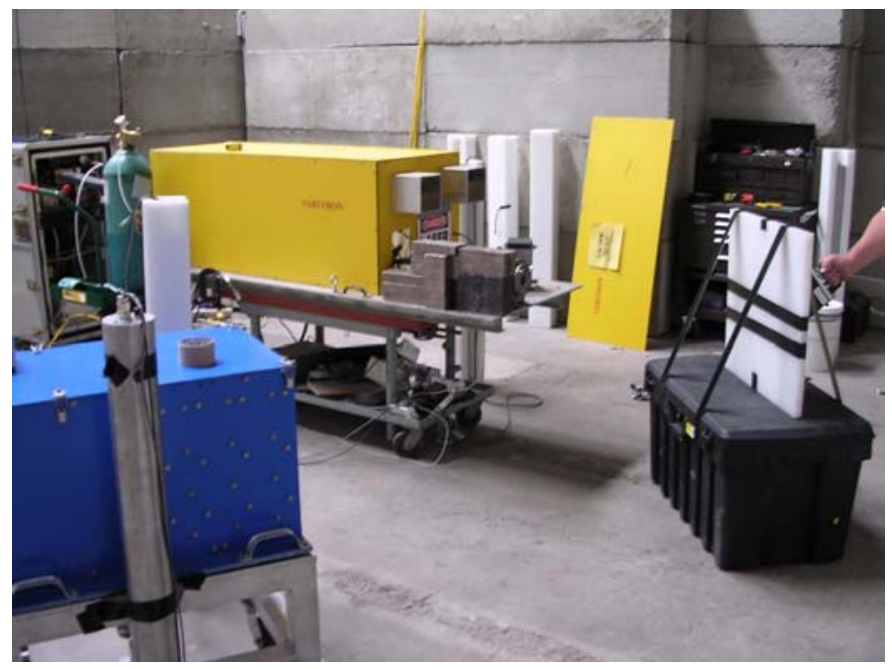

Fig. 6. General experimental setup.

\section{Resulting IMAGES}

Several different experiments were performed which included varying the accelerator energies, accelerator pulse rates, acquisition window, target materials, and distances. A summary of the important observations and experiments and the resulting data follows.

An experiment was performed with polyethylene alone, without heavy metal in the target. Very few neutrons (fast or thermal) were detected in that case. When heavy metals were placed in the photon beam, few neutrons were produced with the electron beam energy below $5-\mathrm{MeV}$, but very high intensities of fast neutrons was detected by the bare INL He-3 tubes. However, no thermal neutron image was obtained unless moderating material was put in close proximity to the target. Using short delay times (1-2 milliseconds) for the data acquisition it was possible to image $\mathrm{W}$, Bismuth and DU targets surrounded by polyethylene, and the signal-tobackground obtained in 10 minute runs was excellent (see Fig. 7 and 8). These images were binned to match the mask pixel size and to get better statistics. The histograms show that of the 361 pixels in the image, which have been rescaled to an intensity range from 1 to 256, there is a narrow distribution of background intensities with a standard deviation of about 18 , and one pixel is about 10 standard deviations. above the background.
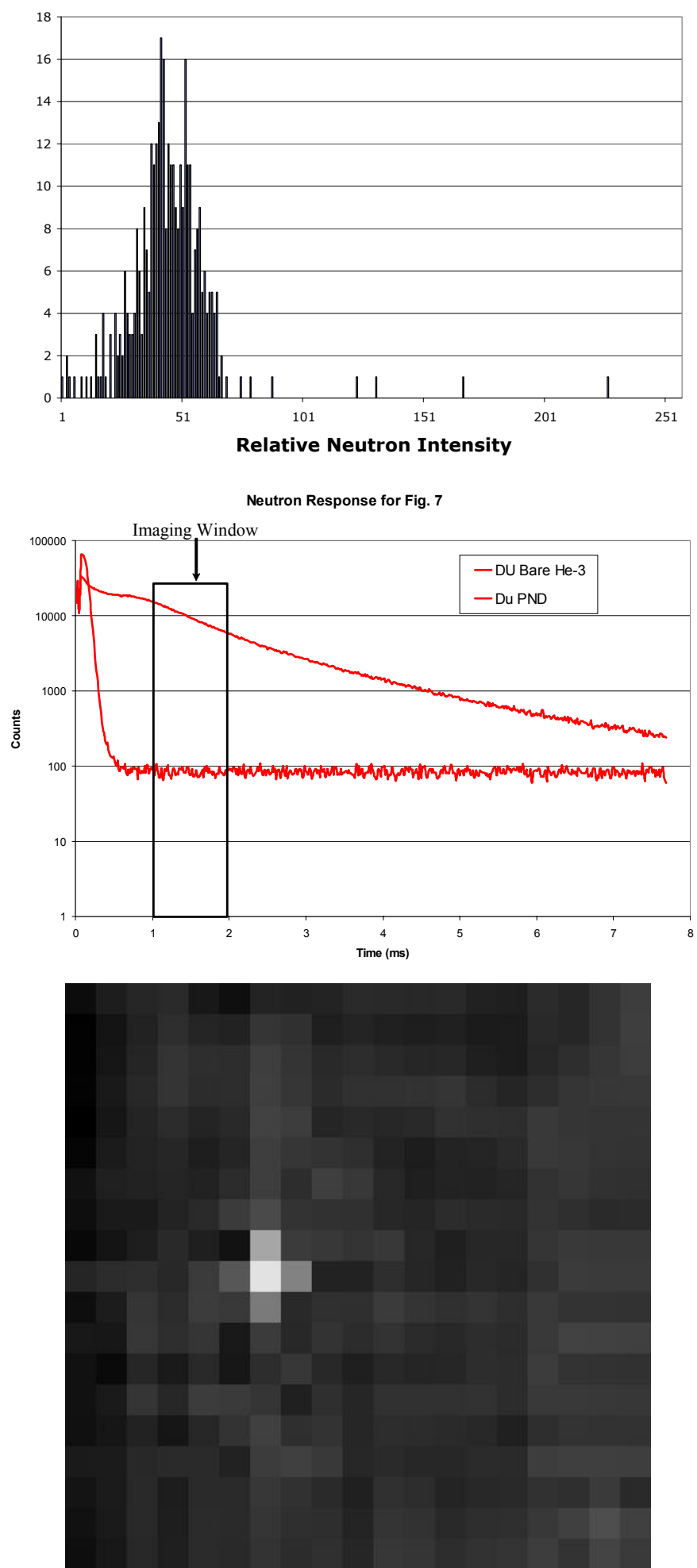

Fig. 7. A histogram of counts for DU in polyethylene, $(1-2 \mathrm{~ms}, 220 \mathrm{k}$ neutrons, mean $=44$, standard deviation $=18$ ) (top). The time-dependent photoneutron environment (middle) and the resulting image (bottom). 


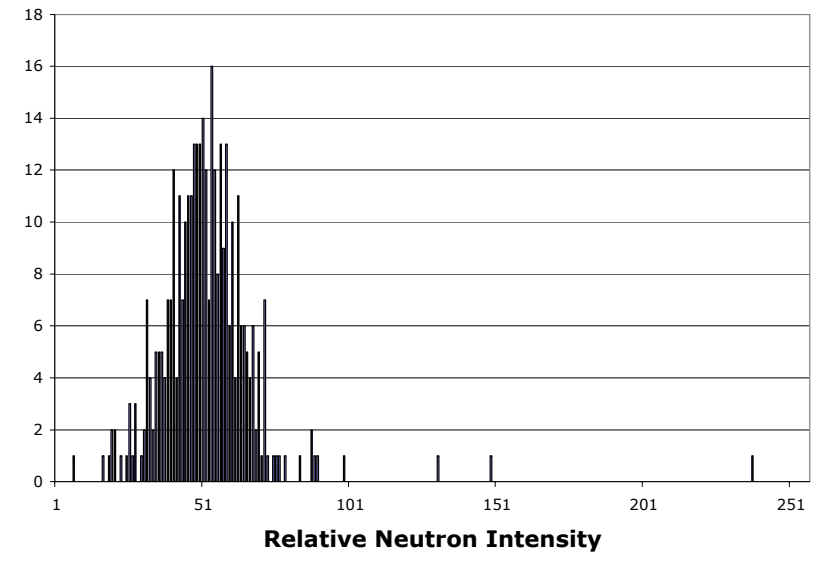

Neutron Response for Fig. 7 and Fig. 8
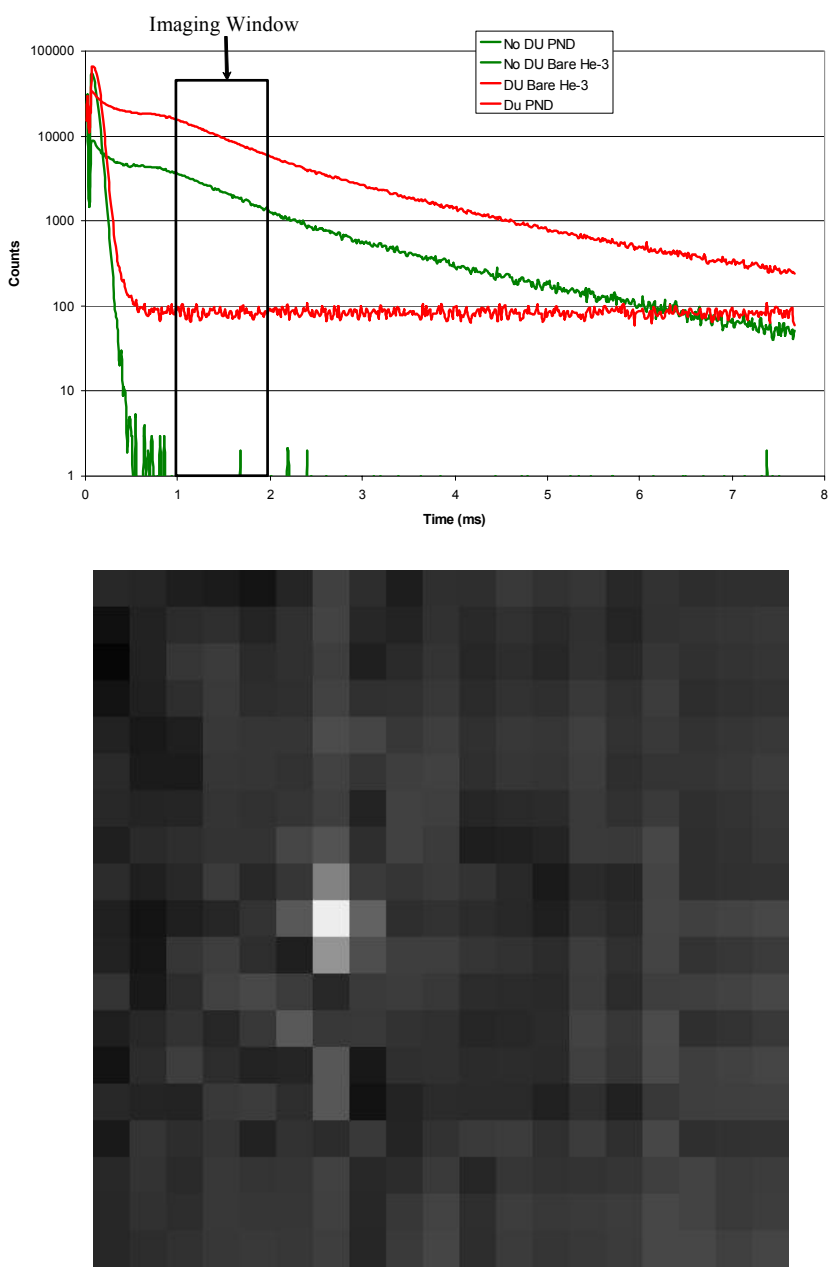

Fig. 8. A histogram of counts for $\mathrm{W}$ in polyethylene, $(1-2 \mathrm{~ms}, 104 \mathrm{k}$ neutrons, mean $=51$, standard deviation $=17$ ) (top). The time-dependent photoneutron environment (middle) and the resulting image (bottom).
By increasing the delay of the acquisition window from 1 to 6.5 milliseconds (Fig. 9) after the photon pulse, it was still possible to image the DU target using the delayed neutrons because they continue to be produced long after the typical "room return" neutrons die-away. In this case, the brightest pixel was about 6 standard deviations above the mean background intensity.

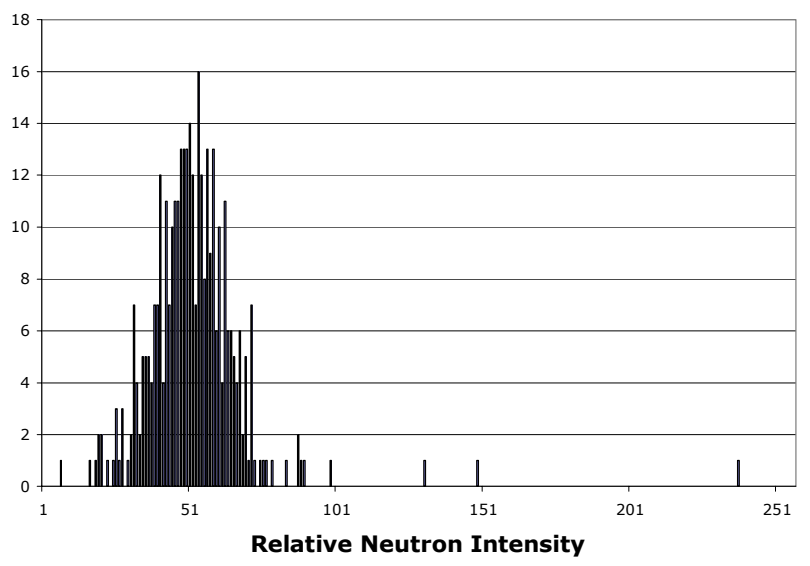

Neutron Response for Fig. 9 and Fig. 10
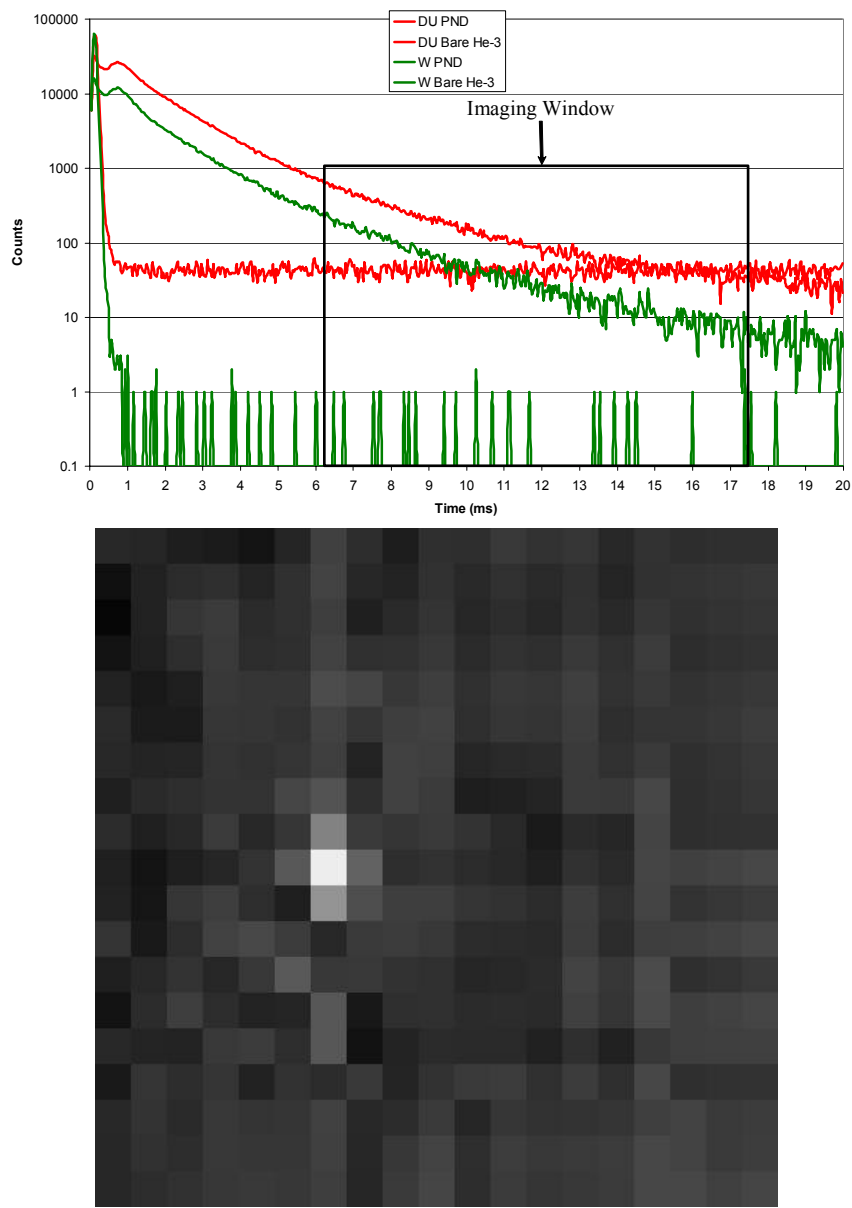

Fig.9. A histogram of counts for DU in polyethylene, $(6.5-17.5 \mathrm{~ms}, 69 \mathrm{k}$ neutrons, mean $=72$, standard deviation $=28$ ) (top). The time-dependent photoneutron environment (middle) and the resulting image (bottom). 
Fig. 10 shows the data acquired with a 6.5 to 17.5 milliseconds acquisition window using a $\mathrm{W}$ target. The count rate was much lower than that with the DU, and the distribution of intensities was essentially random.
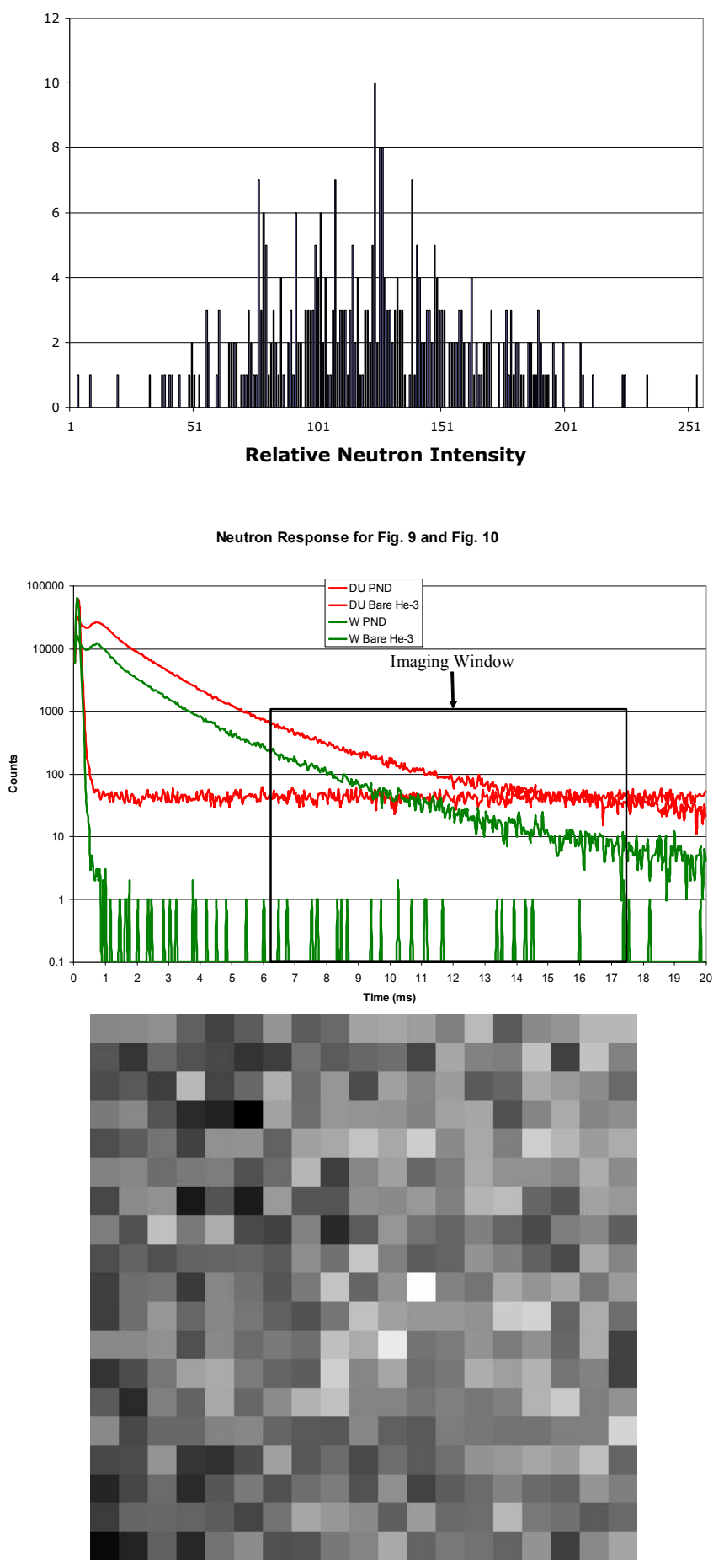

Fig. 10. A histogram of counts for $\mathrm{W}$ in polyethylene, $6.5-17.5 \mathrm{~ms}, 17 \mathrm{k}$ neutrons, mean $=122$, standard deviation $=41$ ) (top). The time-dependent photoneutron environment (middle) and the resulting image (bottom).
Although there is always one pixel in the image that appears to be the brightest, it is only 3 standard deviations above the mean, and could belong to the background population of pixel intensities. Also, in this case the brightest pixel is not located at the source position. Therefore, the delayed acquisition window allows a distinction to be made between DU and W targets.

\section{CONCLUSIONS AND RECOMMENDATIONS}

With the three heavy metals tested, large numbers of photoneutron reactions were excited, injecting fast neutrons into the adjacent polyethylene. These neutrons thermalized in the polyethylene and took a few milliseconds to die away, during which time it was possible to form an image. For gated data acquisitions with delays longer than 6.5 milliseconds, the prompt neutrons were essentially gone, but the delayed photofission neutrons were still being generated by the DU. Hence, thermal neutron imaging can be used in combination with a high energy photon source to locate a dense, heavy metal object in contact with hydrogenous material. By adjusting the delay, it is possible to distinguish nuclear material (fissionable) from non-fissionable metals.

There are several recommendations that may further enhance the imaging system's capability in active environments which include:

1. Reducing the linac pulse rate, while maintaining the average beam current. This would allow longer acquisition windows.

2. Increasing the linac energy above $10-\mathrm{MeV}$. This would produce more delayed neutrons for the same beam current.

3. Increase sensitivity to delayed photofission neutrons. This would provide more neutron counts in the imaging system from the nuclear material.

4. Decreasing the sensitivity to the "room return" neutrons. This would enable counting nearer the photon pulse.

5. Generate time-dependent image data. This would enable the acquisition window to be optimized after the data was collected.

6. Provide real-time image development. This would enable the data to be assessed as it is being acquired.

7. Lower the grid voltage to allow quicker recovery from the photon pulse.

\section{REFERENCES}

[1] J. L. Jones, "Pulsed Photoneutron Interrogation: The GNT Demonstration System," INEL Report WINCO-1225, October 1994.

[2] J. L. Jones, "Photofission-based, Nuclear Material Detection: Technology Demonstration," INEEL Report INEEL/EXT-02-014406, December 2002.

[3] P. E. Vanier, "Improvements in coded thermal neutron imaging," Brookhaven Report BNL-71468-2003-CP, 2003. 